\title{
Articuler les temps composés de l'économie
}

Dominique Margairaz et Philippe Minard

\section{(2) OpenEdition}

Journals

Édition électronique

URL : https://journals.openedition.org/ahrf/11186

DOI : $10.4000 /$ ahrf.11186

ISSN : 1952-403X

Éditeur :

Armand Colin, Société des études robespierristes

Édition imprimée

Date de publication : 1 juin 2008

Pagination : 3-4

ISBN : 978-2200-92514-7

ISSN : 0003-4436

Référence électronique

Dominique Margairaz et Philippe Minard, «Articuler les temps composés de l'économie », Annales historiques de la Révolution française [En ligne], 352 I avril-juin 2008, mis en ligne le 18 décembre 2009, consulté le 23 avril 2022. URL : http://journals.openedition.org/ahrf/11186 ; DOI : https://doi.org/ 10.4000/ahrf.11186 


\section{ARTICULER LES TEMPS COMPOSÉS DE L'ÉCONOMIE}

Dominique MARGAIRAZ, Philippe MINARD

$\mathrm{Au}$ moment du Bicentenaire, François Hincker dénonçait l'idée reçue d'une longue prédominance de l'histoire économique dans les études révolutionnaires. Dressant le bilan des recherches, il soulignait le poids de nos ignorances, et l'importance des chantiers à ouvrir ou réouvrir. Près de vingt ans après, l'histoire économique reste-t-elle un parent pauvre de l'historiographie révolutionnaire ? Nous ne le croyons pas, même si les travaux n'ont pas, en ce domaine, la même visibilité que dans d'autres secteurs. D'où l'idée de ce numéro spécial destiné à rendre plus sensibles les pistes suivies, et à envisager de nouvelles perspectives.

Cette question de la visibilité tient en fait à une difficulté spécifique, qui est celle des temporalités. L'histoire économique de la Révolution est en effet tiraillée entre deux pôles. D'un côté, la période révolutionnaire, si riche en événements de tous ordres, est souvent examinée en elle-même et pour elle-même, sous l'empire de l'exceptionnel : l'attention est portée aux fluctuations de la conjoncture, ou bien aux revirements et effets des politiques économiques menées par les différents gouvernements, avec plus ou moins de succès.

D'un autre côté, quand l'attention est portée aux dynamiques de plus longue durée, les études tendent à « enjamber » la séquence révolutionnaire, à l'isoler comme une phase " anormale ", et au mieux, se contentent d'enregistrer le remodelage des configurations institutionnelles. Ainsi, la problématique de la croissance de l'économie française tend souvent à ranger la Révolution au rang des accidents qui ont « handicapé » la France, lui ont fait prendre du « retard »...

Bref, nous butons sur la vieille question des ruptures et continuités, qui peut paraître triviale, mais s'avère en vérité centrale. Avec, en arrière- 
fond, une autre question : celle de l'urgence, du pilotage à vue qu'ont connu bien des acteurs en ces temps d'incertitude accrue.

Or, si l'on veut bien prendre au sérieux la question: « qu'est-ce que la Révolution française a changé ? » dans le domaine de l'économie, il nous faut rompre l'alternative réductrice décrite plus haut, et repenser les emboîtements des diverses temporalités économiques. Ceci d'autant plus que le changement de focale chronologique permet aussi, en sens inverse, de relire l'économie du XVIII ${ }^{e}$ siècle, en décentrant le regard : on aperçoit mieux ce qui soutenait les équilibres ou les tendances, structures et mouvements anciens, si l'on examine la situation une fois ceux-ci disparus ou malmenés.

D'où l'idée de sélectionner un certain nombre de thèmes ou d'objets, en demandant pour chacun : quelle est la bonne focale, quelle temporalité faut-il envisager pour apercevoir les changements, repérer les discordances majeures, et comprendre comment les uns et les autres s'articulent aux autres phénomènes, aux autres composantes de l'économie?

Il est clair qu'un certain nombre de ruptures sont engagées dans les années 1760 ou 1770, et que 1791 n'en marque pas moins, à l'évidence, un tournant majeur. Mais la reconfiguration d'ensemble opérée alors signifie-t-elle une tabula rasa complète ? Les différents secteurs n'obéissent bien sûr pas à une temporalité uniforme : c'est cet emboîtement de temporalités, d'ondes de choc différenciées, avec des phénomènes d'inertie plus ou moins forts, des contraintes héritées du chemin suivi (path dependency) également, qui doit être restitué.

Dans ce but, ce numéro rassemble des études de statuts divers (bilan sectoriel ou étude de cas), mais toutes guidées par une question sous-jacente : quelle est l'échelle temporelle pertinente pour le thème ou le secteur étudié ?

IDHE-CNRS UMR 8533

Université Paris 1

17 rue de la Sorbonne 75005 Paris dmargairaz@wanadoo.fr
IDHE-CNRS UMR 8533, et EHESS Université Paris 8 Département d'histoire 2 rue de la liberté 93526 Saint-Denis cedex philippe.minard@ens.fr 